\title{
Self reported musculoskeletal symptoms in the neck/ shoulders and/or arms and general health (SF-36): eight year follow up of a case-control study
}

\section{A Nordlund, K Ekberg}

See end of article for authors' affiliations

Correspondence to: Dr A Nordlund, Department of Health and Society, Faculty of Health Sciences, National Centre of Work and Rehabilitation, Linköping, SE-581 85, Sweden; anders.nordlund@ ihs.liu.se

Accepted 8 September 2003

\begin{abstract}
Aims: To explore and compare the prevalence after eight years of self reported musculoskeletal symptoms and general health (SF-36) for groups with initially different degrees of severity of symptoms in the neck/ shoulders and/or arms.

Methods: A case-control study was performed in 1989 comprising 129 clinically examined cases and 655 survey controls. The study population was followed up in 1997 with a postal survey. The controls, none of which were clinically examined at baseline (1989), were divided into groups according to degree of severity of self reported symptoms in the neck/shoulders and/or arms at baseline: no symptoms, light symptoms, and severe symptoms. Cases were clinically diagnosed with a musculoskeletal disorder of the neck/shoulders and/or arms at baseline.

Results: At the 1997 follow up, there was a trend of increasing prevalence of musculoskeletal symptoms, as well as decreasing health status as rated in the SF-36 over the three severity groups among controls. Only small differences were seen between the cases and the controls reporting severe musculoskeletal symptoms or the neck/shoulders and/or arms.

Conclusion: The degree of questionnaire based self reported musculoskeletal symptoms of the neck/ shoulders and/or arms clearly indicate different degrees of future health problems (both in terms of self reported musculoskeletal problems and health in general as captured by the SF-36). Therefore, there is a need for improved intervention and health promotion strategies. Such effort should be implemented before musculoskeletal symptoms have developed to clinical cases, particularly in the realm of the workplace.
\end{abstract}

D uring the 1990s, long term sick-leave has increased in Sweden. ${ }^{1}$ Health complaints relating to musculoskeletal pain and discomfort are the most common occupationally related illnesses ${ }^{2}$ and about $40 \%$ of all sickleaves in Sweden are currently due to musculoskeletal problems. ${ }^{1}$ Thus, in addition to the suffering of the individual, musculoskeletal problems incur large societal costs as they cause long periods of sick leave involving treatment and rehabilitation. ${ }^{3}$

Population prevalence estimates of musculoskeletal problems of the neck/shoulder, hand/arm, and upper back have varied widely. Examples of reported prevalence are $20 \%$ in Sweden in $1997,{ }^{4} 20-40 \%$ in the Netherlands, ${ }^{5}$ and $38 \%$ in the British population, ${ }^{6}$ but the comparability of the figures is questionable as case definition and study methodology varied substantially. It seems however, that the largest share of musculoskeletal problems is of the neck/shoulders or upper limb area. $^{7}$ Although research regarding musculoskeletal problems has been extensive, most studies have been crosssectional and the lack of longitudinal data has often been pointed out. ${ }^{289}$ As indicated by the high prevalence figures, musculoskeletal problems are a very common health complaint. Therefore, to better direct resources tackling this problem, the level of detail of musculoskeletal problems needs expansion, since they can be described as a continuum from mild symptoms to very severe and incapacitating disorders. ${ }^{10}$ The development and long term prognosis for individuals with different degrees of severity of musculoskeletal disorders has not been studied extensively, but there are indications that early musculoskeletal symptoms predispose for a worse prognosis. Studies have shown that a history of musculoskeletal symptoms has been associated with future symptoms, ${ }^{11-14}$ that baseline complaints and history of sickness absence due to musculoskeletal problems predicted future sickness absence due to musculoskeletal problems, ${ }^{15}$ that greater self reported pain and functional disability predicted prolonged disability, ${ }^{16}$ and, that empirically derived subgroups with different degree of musculoskeletal pain also differed in clinical course. ${ }^{17}$ Furthermore, while studies have shown that a large proportion of individuals with low back pain will recover without medical treatment, ${ }^{18}$ less is known regarding the recovery rate for disorders in the neck and shoulders. ${ }^{14}$

A case-control study on musculoskeletal disorders of the neck/shoulders and/or arms from $1989^{19}$ was followed up in 1997. The aim of the present study is to describe the prevalence of self reported musculoskeletal symptoms and self rated health (SF-36) at follow up in 1997 for cases diagnosed in 1989 (baseline), and population controls with different severity of self reported musculoskeletal symptoms of the neck/shoulders and/or arms at baseline. Of particular interest from the perspective of prevention and intervention is to elucidate the prognostic potential of self reported symptoms regarding future symptoms. Self rated health has proven to be an important prognostic factor for morbidity and mortality ${ }^{2021}$ and is included as a marker of the perceived impact of pain and disorders on the individual's general health and health related quality of life.

\section{METHODS}

The case-control study population that is now followed up for eight years, has been described in detail previously. ${ }^{19} 22$ In brief, the study was performed in a semi-rural community in southern Sweden. Selected subjects were aged between 18 
Main messages

- Different degrees of severity of self reported musculoskeletal symptoms are indicative of future health problems in terms of musculoskeletal problems as well as health in general (SF-36).

and 59 years at baseline in 1989. They had at least two months occupational experience of the last job held, were not employed by the large rubber industry in the area, and had not been on longer (two months) sick-leave during the past six months. The cases were recruited consecutively during a period of 18 months (August 1988 to October 1989) among those consulting the occupational health care units in the community for musculoskeletal disorders in the neck, shoulders, and/or arms. Cases were excluded if previously continuous sick-leave for the disorder in question exceeded four weeks, the disorder was caused by trauma, bacterial agents, or by an accident, they had developed malignancies, rheumatic diseases, or drug abuse, or become pregnant. The same physician and physiotherapist examined all 129 cases. Diagnoses were based on the predefined criteria described by Waris and colleagues. ${ }^{23}$

The control group consisted of a stratified random sample of about $10 \%$ of the working population from which the cases had been recruited $(\mathrm{n}=900)$. Due to limited funds, controls were not examined clinically. At baseline (1989), 655 controls returned the questionnaire; 18 did not belong to the active labour force and were excluded, giving a response rate of $74 \%$. At follow up in 1997, the questionnaire was sent by mail to the 129 cases and the 637 controls that had responded to the baseline questionnaire. Eighty cases and 457 controls responded, giving response rates of $62 \%$ and $72 \%$, respectively. For the purpose of the following analyses, one case was excluded since in spite of having the diagnosis epicondylitis she did not report any musculoskeletal symptoms at all in the baseline questionnaire.

\section{Musculoskeletal symptoms}

At both baseline and follow up, all subjects answered a modified version of the Nordic questionnaire ${ }^{24}$ on musculoskeletal symptoms covering 10 different anatomical sites. For each anatomical site, subjects stated whether they had had symptoms during the past six months. These 10 anatomical sites were subsequently combined to five body areas as they are strongly interrelated: neck/shoulders, arms (including elbows/arms/wrists), upper back, lower back, and legs (including hips/knees/ankles). Eleven subjects had partial missing data regarding musculoskeletal symptoms; these were grouped based on answers regarding other anatomical sites within the same body area (that is, missing answers were regarded as no symptoms in that particular anatomical site). Fourteen subjects had completely missing data for one or more body areas; in these instances no imputations were made.

Self reported symptoms in the neck/shoulders and/or in the elbows/arms/hands were classified according to severity. Symptoms reported as being severe or as having interfered with work were regarded as "severe". Results will be presented by severity of musculoskeletal symptoms of the neck/shoulders and/or elbows/arms/hands using baseline data, for the following four groups: controls reporting no symptoms, controls reporting light symptoms, controls reporting severe symptoms, and cases. Cases were treated as a separate group since they were recruited by a different method (case ascertainment rather than being part of the
Policy implications

- There is a need for improved early strategies of intervention and health promotion at the workplace before clinical symptoms have developed.

random sample) and had a diagnosed musculoskeletal disorder.

\section{Self rated health}

In the follow up questionnaire, the Medical Outcome Study 36-Items Short Form (SF-36) ${ }^{25}{ }^{26}$ was added. The SF-36 is a health survey consisting of 36 items, which generates a health profile of eight subscale index values. ${ }^{26}$ The subscales, all ranging from 0 (worst) to 100 (best), are: physical functioning (PF, consisting of 10 items), role limitations due to physical problems ( $\mathrm{PF}$, four items), pain (BP, two items), general health perceptions ( $\mathrm{GH}$, five items), vitality/energy (VT, four items), social functioning (SF, two items), role limitations due to emotional problems (RE, three items), and mental health ( $\mathrm{MH}$, five items). The final item, which was not used here, deals with health transition during the previous year.

\section{Employment status}

As the follow up period is rather long, eight years, both cases and controls grew older and many changed their employment status between baseline (1989) and follow up (1997). Most importantly, a number of persons reported, in addition to age related retirement, early retirement (due to ill health) or being on sick-leave at follow up. Though not necessarily related to musculoskeletal problems, such data indicate potentially severe health problems in the different groups and may affect comparisons of self reported musculoskeletal symptoms and health. Therefore, data on self reported employment status at follow up are presented before the prevalence and health (SF-36) data. Also, the influence of such changes in employment status will be assessed in a sensitivity analysis where prevalences of musculoskeletal disorders are compared only for those still in the workforce (for example, employed or unemployed).

\section{Data analyses}

Because of the descriptive nature of this study, results are presented in the form of prevalence rates and mean values (SF-36). The focus here is on the outcome for groups of musculoskeletal symptoms of different severity at baseline, and as the number of male cases was low $(n=19)$ no gender specific analyses were carried out. Also, as the age distribution of the four groups under study was similar, no age adjustment was deemed necessary.

Comparisons of group means (proportions) were carried out using the two-sample $t$ test (Pearson's $\chi^{2}$ ). Tests for trend over symptom severity categories were carried out using simple linear regression analysis $\left(\chi^{2}\right.$ test for trend). Probability values of 0.05 or lower were considered statistically significant. SPSS version 11.5.1 was used in all analyses.

Adjustment for the impact of factors such as gender and immigration status could be argued for from a confounding perspective; in particular, since these groups are known to differ with respect to prevalence and risk of musculoskeletal disorders and the proportion of women and immigrants were higher in the case than in the control group. However, as the aim of this study was to describe and compare groups of different severity baseline with respect to outcomes at follow 
up and as adjustment for such factors would reduce the impact of the "natural" mix of risk categories on outcome differences, it was decided not to adjust comparisons.

\section{RESULTS}

\section{Responders versus non-responders}

The responders and non-responders at follow up in 1997 could be compared using the baseline information obtained in 1989. The percentage of immigrants was higher among non-responders than among responders, $41 \%$ versus $23 \%$ among cases and $19 \%$ versus $11 \%$ among controls $(p<0.05$ in both instances). Also, in the control group, the percentage reporting overtime work was lower among non-responders $(46 \%)$ than responders $(55 \%, \mathrm{p}<0.05)$ and the percentage smokers tended to be higher among non-responders (33\%) than responders $(25 \%, \mathrm{p}=0.07)$. It should be noted that as the number of cases was rather low (79 responders and 49 non-responders) it was difficult to assess the differences; however, numerical differences were generally rather small. Finally, only small and statistically non-significant differences in the prevalence of self reported musculoskeletal symptoms at baseline were seen between responders and non-responders.

\section{Demographic and background characteristics}

Table 1 gives background characteristics for cases and controls responding to the follow up survey in 1997. The percentages of women and immigrants were higher among cases compared with controls. At baseline as well as follow up, the percentage of smokers was higher among cases than controls and the percentage reporting regular overtime work was higher among controls than cases both at baseline and at follow up. The percentage of single households decreased and the percentage having children under 7 years of age increased between baseline and follow up, particularly in the control group.

\section{Employment status at follow up}

At the follow up in 1997, 65.8\% of the cases and $71.8 \%$ of the controls reporting severe symptoms in the neck/shoulders and/or arms at baseline were still gainfully employed (table 2). The corresponding figures among the controls reporting none or light symptoms in the neck/shoulders and/ or arms baseline, were $87.3 \%$ and $88.4 \%$ respectively. Although the numbers were rather small, there was a tendency for the cases and the controls reporting severe symptoms in the neck/shoulders and/or arms at baseline, to be on sick-leave or having retired for reasons of ill-health (part or full time) at follow up to a larger extent than the other two groups.
Self reported musculoskeletal symptoms baseline and follow up

Cases had a diagnosed musculoskeletal disorder in the neck/ shoulders and/or arms at baseline and were not classified according to baseline severity of self reported symptoms. The majority $(86.1 \%)$ of cases reported severe symptoms at baseline. At follow up, this figure had decreased to $67.5 \%$ and by then, six of the 79 cases $(7.8 \%)$ did not report any musculoskeletal symptoms in the neck/shoulders and/or arms (table 3 ).

In the control group as a whole, the percentage reporting severe musculoskeletal symptoms in the neck/shoulders and/ or arms increased from 17.1 to 27.5, between baseline (1989) and follow up (1997), while the percentage reporting no such symptoms decreased from 43.1 to 34.9 .

Among the controls reporting severe musculoskeletal symptoms in the neck/shoulders and/or arms at baseline, the distribution on severity categories at follow up (1997) was similar to the cases (table 3 ). The majority (65.8\%) still reported severe symptoms in the neck/shoulders and/or arms at follow up, and $9.2 \%$ (seven individuals) did not report any symptoms at all in the neck/shoulders and/or arms at follow up. In the group of controls who reported light symptoms in the neck/shoulders and/or arms at baseline, $25.8 \%$ reported severe symptoms and $22.0 \%$ reported no symptoms at follow up. Finally, table 3 shows that out of a group of symptomfree individuals, $43.1 \%$ reported musculoskeletal symptoms of the neck/shoulders and/or arms at follow up eight years later.

Table 4 shows the baseline and follow up prevalence of self reported symptoms in the upper and lower back and legs in the three subgroups among controls and the group of cases. Controls not reporting any symptoms in the neck/shoulders and/or arms at baseline consistently reported the lowest prevalence: $5.1 \%, 35.0 \%$, and $32.0 \%$ for upper back, lower back, and legs respectively at baseline with the corresponding figures at follow up being: $15.2 \% 36.0 \%$, and $32.5 \%$. Cases reported the highest prevalence of upper back symptoms (54.4\% and $58.2 \%$ at baseline and follow up, respectively). Controls reporting severe symptoms in the neck/shoulders and/or arms at baseline reported the highest prevalence of symptoms in the lower back and legs at baseline $(62.8 \%$ and $66.7 \%$, respectively) and follow up $(65.4 \%$ and $64.1 \%$, respectively). As indicated by the $95 \%$ confidence intervals, there was evidence of a trend in prevalence over the three different control groups, both at baseline and follow up $(\mathrm{p}<0.001)$. However, the $95 \%$ confidence intervals overlapped in all instances between the cases and the controls reporting severe symptoms in the neck/shoulders and/or arms at baseline.

Regarding changes in prevalence over time, self reported symptoms of the upper back apparently increased between

Table 1 Description of cases and controls at baseline and follow up

\begin{tabular}{|c|c|c|c|c|c|c|}
\hline & \multicolumn{3}{|l|}{ Baseline (1989) } & \multicolumn{3}{|l|}{ Follow up (1997) } \\
\hline & Controls $(n=457)$ & Cases $(n=79)$ & $p$ value & Controls $(n=457)$ & Cases $(n=79)$ & $p$ value \\
\hline Age, mean (SD) & $37.8(11.2)$ & $39.3(10.7)$ & 0.249 & $45.8(11.2)$ & $47.3(10.7)$ & 0.249 \\
\hline BMI, mean (SD) & $23.9(3.3)$ & $23.3(3.8)$ & 0.187 & $25.2(3.9)$ & $24.6(3.5)$ & 0.160 \\
\hline Over weight (BMI > 25), n (\%) & $136(30.3)$ & $21(26.9)$ & 0.548 & $217(48.3)$ & $36(46.8)$ & 0.798 \\
\hline Women, $\mathrm{n}(\%)$ & $216(47.3)$ & $60(75.9)$ & $<0.001$ & & & \\
\hline Born abroad, n (\%) & $51(11.2)$ & $18(22.8)$ & 0.004 & & & \\
\hline Single households*, $n(\%)$ & $125(27.7)$ & $15(19.0)$ & 0.107 & $85(18.6)$ & $13(16.5)$ & 0.649 \\
\hline Smoker, n (\%) & $116(25.4)$ & 35 (44.3) & 0.001 & $92(20.6)$ & $30(38.5)$ & 0.001 \\
\hline Alcohol abstainers, n (\%) & $80(17.9)$ & $16(20.3)$ & 0.617 & $67(15.3)$ & $16(20.5)$ & 0.248 \\
\hline Children $<7$ years, $\mathrm{n}(\%)$ & 88 (19.5) & $18(22.8)$ & 0.496 & $136(29.8)$ & $21(26.6)$ & 0.567 \\
\hline Daytime workers, $\mathrm{n}(\%)$ & $367(80.7)$ & $65(82.3)$ & 0.735 & $303(71.6)$ & $38(62.3)$ & 0.135 \\
\hline Part time workers, $n$ (\%) & $113(25.3)$ & $25(31.6)$ & 0.236 & $107(28.1)$ & $15(30.6)$ & 0.712 \\
\hline
\end{tabular}


Table 2 Employment status at follow up, \% (n)

\begin{tabular}{|c|c|c|c|c|c|}
\hline & \multicolumn{3}{|l|}{ Controls } & \multirow[b]{2}{*}{ Cases } & \multirow[b]{2}{*}{ p value } \\
\hline & No symptoms & Light symptoms & Severe symptoms & & \\
\hline Working status & $n=197$ & $n=181$ & $n=78$ & $\mathrm{n}=79$ & \\
\hline In work & $87.3(172)$ & $88.4(160)$ & $71.8(56)$ & $65.8(52)$ & $<0.001^{*}$ \\
\hline Unemployed & $3.0(6)$ & $1.7(3)$ & $3.8(3)$ & $6.3(5)$ & \\
\hline Retired (age) & $3.0(6)$ & $3.3(6)$ & $5.1(4)$ & $2.5(2)$ & \\
\hline $\begin{array}{l}\text { Retired due to ill health } \\
\text { (part or full time) }\end{array}$ & $3.0(6)$ & $1.7(3)$ & $10.3(8)$ & $19.0(15)$ & \\
\hline Other & $3.5(7)$ & $5.0(9)$ & $8.9(7)$ & $6.3(5)$ & \\
\hline
\end{tabular}

baseline and follow up among the three groups of controls. Among cases, the prevalence of self reported symptoms in the legs increased from $46.8 \%$ to $59.5 \%$ between baseline and follow up. In all other instances, changes in prevalence over time were rather small given the small numbers involved.

\section{SF-36 at follow up}

As fig 1 shows, the best health in terms of the SF-36 was seen among the controls not reporting any symptoms in the neck/ shoulders and/or arms at baseline, and the poorest health among controls reporting severe symptoms in the neck/ shoulders and/or arms at baseline and the cases. The largest differences were seen in the SF-36 subscales capturing physical aspects of health (PF, RP, and BP), the general health subscale $(\mathrm{GH})$, and the subscale measuring energy/ vitality (VT). While none of the differences between the controls reporting severe symptoms in the neck/shoulders and/or arms at baseline and the cases was statistically significant, there was evidence of a trend in self reported health (SF-36) at follow up over the three different control groups $(p<0.001$ for all but role emotional (RE) for which $\mathrm{p}=0.002)$.

\section{DISCUSSION}

In this study, a cohort of cases (with diagnosed musculoskeletal disorders of the neck/shoulder and/or arms) and population controls (classified into three groups of increasing severity based on self reported symptoms in the neck/ shoulder and/or arms at baseline) were followed up for eight years with regard to self reported musculoskeletal symptoms and self reported health (SF-36). It was clear that the case group and the group of controls reporting severe musculoskeletal symptoms in the neck/shoulders and/or arms at baseline were worst off in terms of prevalence of self reported musculoskeletal symptoms and self rated health (SF-36) at follow up (1997). These findings are in line with other studies indicating that a history of musculoskeletal symptoms involving a degree of disability implies higher risk for persistency or recurrence of symptoms and thus a lower chance of recovery. ${ }^{11-13} 1727$

Regarding the SF-36, the baseline, self reported symptoms based subgroup classification of controls manifested distinct differences in terms of health as measured by the SF-36 at follow up eight years later (fig 1). This gradient, though most clearly manifested in the physical health related subscales ( $\mathrm{PF}, \mathrm{RP}$, and $\mathrm{BP}$ ) was also present the in the overall subscale (GH) and the mental health related subscales (VT, SF, RE, and $\mathrm{MH}$ ). It thus seems as if early musculoskeletal symptoms as perceived by the individual may be of prognostic importance not only with regard to future prevalence of musculoskeletal symptoms, but also with regard health in more general terms.

Self rated health and quality of life may be a consequence of the degree of musculoskeletal symptoms, but may also affect the prognosis on work ability. ${ }^{28}{ }^{29}$ During the eight year follow up employment status changed and the proportion still gainfully employed were lowest among cases and controls reporting severe musculoskeletal symptoms in the neck/shoulders and/or arms at baseline. Although numbers were small, the proportions retired due to sickness at follow up were highest in these groups.

It should also be noted that, apart from the rather dismal overall picture concerning cases and controls reporting severe musculoskeletal symptoms in the neck/shoulders and/or arms at baseline, there was also evidence of some improvements. The absolute percentages may seem rather small, 7.8\% and $9.2 \%$, respectively, but it should be emphasised that these subjects did not report any musculoskeletal symptoms in the neck/shoulders and/or arms at follow up.

The subgroup classification used here was based on two sources of information. While the cases had diagnosed disorders established during clinical examination, the control group was subdivided into three groups of different severity using their answers to questions regarding musculoskeletal symptoms. The division was based on whether controls reported symptoms having interfered with work or whether they reported their symptoms as light or severe. There were other questions reflecting the severity of reported symptoms in terms of how often symptoms were experienced and the current degree of pain experienced. Although these questions related to self reported musculoskeletal symptoms in general, answer patterns closely followed the implemented severity classification of controls based on site specific questions, with high percentages reporting frequent and strong pain among the controls reporting severe symptoms at baseline. Ohlsson and colleagues $^{30}$ compared findings of musculoskeletal

Table 3 Self reported symptoms neck/shoulders and arms at follow up

\begin{tabular}{lllll}
\hline $\begin{array}{l}\text { Degree of self reported } \\
\text { symptoms of the neck/ } \\
\text { shoulders and/or arms }\end{array}$ & $\begin{array}{l}\text { Controls, no } \\
\text { symptoms }(\mathbf{n = 1 9 7 )}\end{array}$ & $\begin{array}{l}\text { Controls, light } \\
\text { symptoms }(\mathbf{n = 1 8 2})\end{array}$ & $\begin{array}{l}\text { Controls, severe } \\
\text { symptoms (n=78) }\end{array}$ & Cases (n=79) \\
\hline No symptoms, \% & $56.9(112)$ & $22.0(40)$ & $9.2(7)$ & $7.8(6)$ \\
Light symptoms, \% & $28.9(57)$ & $52.2(95)$ & $25.0(19)$ & $24.7(19)$ \\
Severe symptoms, \% & $14.2(28)$ & $25.8(47)$ & $65.8(50)$ & $67.5(52)$ \\
\hline
\end{tabular}


Table 4 Prevalence (95\% confidence intervals) of musculoskeletal symptoms in other body parts

\begin{tabular}{lllll}
\hline $\begin{array}{l}\text { Self reported } \\
\text { symptoms }\end{array}$ & $\begin{array}{l}\text { Controls, no } \\
\text { symptoms }(\mathbf{n}=197)\end{array}$ & $\begin{array}{l}\text { Controls, light } \\
\text { symptoms }(\mathbf{n}=182)\end{array}$ & $\begin{array}{l}\text { Controls, severe } \\
\text { symptoms (n=78) }\end{array}$ & Cases (n=79) \\
\hline $\begin{array}{l}\text { At baseline (1989) } \\
\text { Upper back }\end{array}$ & $5.1(2.0: 8.1)$ & $23.1(17.0: 29.2)$ & $43.6(32.6: 54.6)$ & $54.4(43.4: 65.4)$ \\
Lower back & $35.0(28.4: 41.7)$ & $55.5(48.3: 62.7)$ & $62.8(52.1: 73.5)$ & $59.5(48.7: 70.3)$ \\
Legs & $32.0(25.5: 38.5)$ & $43.4(36.2: 50.6)$ & $66.7(56.2: 77.1)$ & $46.8(35.8: 57.8)$ \\
& & & & \\
At follow up (1997) & $15.2(10.2: 20.2)$ & $33.0(26.1: 39.8)$ & $53.8(42.8: 64.9)$ & $58.2(47.4: 69.1)$ \\
Upper back & $36.0(29.3: 42.7)$ & $60.4(53.3: 67.5)$ & $65.4(54.8: 75.9)$ & $59.5(48.7: 70.3)$ \\
Lower back & $32.5(25.9: 39.0)$ & $46.7(39.5: 54.0)$ & $64.1(53.5: 74.7)$ & $59.5(48.7: 70.3)$ \\
Legs & & & & \\
\hline
\end{tabular}

complaints in the neck and upper limbs in clinical examination with self reported symptoms in the modified version of the Nordic questionnaire. ${ }^{30}$ A reasonable agreement with respect to findings on a more general level (for example, neck/shoulders as opposed to neck and shoulders considered as separate entities) were found. As the controls were not clinically examined, one may therefore suspect that among those controls reporting severe symptoms at baseline an unknown proportion probably had musculoskeletal problems in their neck/shoulders and/or arms that would have fulfilled diagnostic criteria, if they had consulted a physician. Reasons for having consulted a physician (cases) or not (severe controls), are not further analysed in the present paper. A possible reason may be differences in working conditions, for example, differences in workload or psychosocial conditions at work between the groups, influencing the need for medical assistance.

Because of the different recruitment of cases and controls (for example, clinical visits and random sample), the two groups also differed with regard to certain demographic and background characteristics. That is, there were more women, current smokers, and immigrants in the case group and, the control group reported working overtime to a larger extent than did cases. These differences, with the exception of immigration status, persisted in a comparison restricted to the controls reporting severe symptoms at follow up and the cases. This pattern is not surprising since it is known that musculoskeletal disorders are more common among women, immigrants, and smokers. ${ }^{4} 1931$ However, whether these groups also are prone for a worse prognosis is unknown. Still in this study, our aim was to describe and compare the outcome at follow up for groups with symptoms of different severity at baseline. Thus, explaining the impact of any risk factor imbalance due to differences in the selection processes

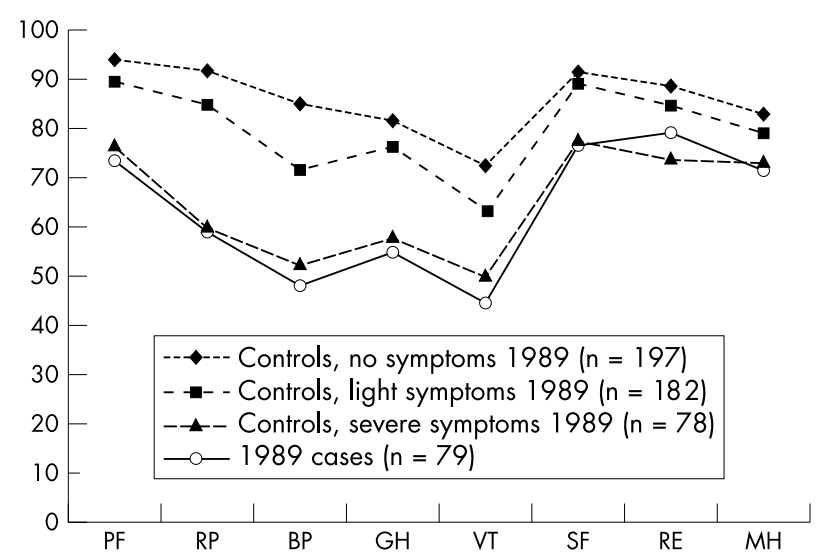

Figure 1 SF-36 in 1997 by symptom category in 1989. of cases and controls goes beyond the scope of this presentation.

The population of cases and controls were eight years older at follow up (1997) than at baseline (1989). One may thus suspect a change in prevalence of musculoskeletal symptoms merely due to individuals getting older, although in a recent review, the association between age and musculoskeletal problems was evident in less than half of the studies including age as a risk factor. ${ }^{9}$ However, calculating prevalence of musculoskeletal symptoms for the age group available at both baseline and follow up (26-56 years) yielded only marginally different prevalence estimates compared with the figures presented in the tables above. Also, employment status changed between baseline and follow up to a different extent in the four groups studied. This may have affected the self report of musculoskeletal problems particularly since the severity of symptoms was based on whether symptoms reported were stated to having interfered with work. The distributions on severity categories at follow up among the cases and controls shown in table 3 as well as the prevalence of other symptoms (table 4) did not change when the analysis was restricted to those still economically active at follow up.

The amount of non-response in this study was considerable, particularly so in the control group, where only 457 $(52 \%)$ of the original 900 controls remained after baseline and follow up surveys. However, apart from immigration status, differences between the responders and the nonresponders in terms of background characteristics and self reported musculoskeletal symptoms at baseline were small and statistically not significant. Although it is difficult to speculate with regard to the effect on results, it may be fair to assume that any bias introduced by non-response probably would not explain the results of this study, in particular since non-responders displayed rather similar prevalence of self reported musculoskeletal symptoms compared to the responders.

Another problem with survey investigations is recall bias; in particular, respondents tend to remember recent events better than more remote events. In this study the recall period for musculoskeletal symptoms was six months, and although respondents may have forgotten to report the oncein-six-months-episode of musculoskeletal problems dating several months back, musculoskeletal problems are chronic and recurrent to its nature. We therefore believe that any such bias is small and that only those that suffer the least are missed.

In summary, questionnaire based self reported musculoskeletal symptoms of the neck/shoulders and/or arms clearly indicate future long term health problems (both in terms of self reported musculoskeletal problems and health in general as captured by the SF-36). It is also clear that subdivision of such self reported symptoms is meaningful in that marked 
differences in health between those reporting no, light, and severe symptoms were seen eight years later. Also, the health of those with severe self reported symptoms of the neck/ shoulders and/or arms classified as severe was similar to those who had a diagnosed musculoskeletal disorder at baseline. These results therefore underscore the need for improved intervention and health promotion strategies, and the need for such effort to be implemented before musculoskeletal symptoms have developed as clinical cases. As musculoskeletal symptoms are known to be related to both ergonomic and psychosocial factors at the workplace, the workplace is the most important arena for such efforts.

\section{ACKNOWLEDGEMENTS}

This work was supported by grants from the Swedish Work Environment Fund.

\section{Authors' affiliations}

A Nordlund, K Ekberg, National Centre of Work and Rehabilitation, Department of Health and Society, Faculty of Health Sciences, National Centre of Work and Rehabilitation, Linköping, SE-581 85, Sweden

\section{REFERENCES}

1 SOU 2000:78. Rehabilitation to work. A reform centering on the individual. Final report from the investigation on work-related rehabilitation. Stockholm, 2000 (in Swedish).

2 Hansson T, Westerholm P, eds. Work and musculoskeletal complaints. Work and Health. Stockholm: NIWL, Stockholm, 2001:12 (in Swedish).

3 National Institute for Occupational Safety and Health. Musculoskeletal disorders and workplace factors. A critical review of the epidemiologic evidence for work-related musculoskeletal disorders of the neck, upper extremity, and low back. Publication no. 97-141. Cincinnati, OH: DHHS (NIOSH), 1997:2.1-5c31.

4 Statistics Sweden. Work Related Problems 1997. Statistiska meddelanden AM 43 SM 9701, Stockholm, 1997 (in Swedish)

5 Health Council of the Netherlands. RSI. Publication No. 2000/22E. The Hague Council of the Netherlands, 2000.

6 Palmer KT, Cooper C, Walker-Bone K, et al. Use of keyboards and symptoms in the neck and arm: evidence from a national survey. Occup Med 2001;51:392-5.

7 Cherry NM, Meyer JD, Chen Y, et al. The reported incidence of work-related musculoskeletal disease in the UK: MOSS 1997-2000. Occup Med 2001;51:450-5.

8 Kristensen TS. Job stress and cardiovascular disease: a theoretic critic review. J Occup Health Psychol 1996; 1:246-60.

9 Malchaire J, Cock N, Vergracht S. Review of the factors associated with musculoskeletal problems in epidemiologic studies. Int Arch Occup Environ Health 2000;74:79-90.
10 Morken T, Riise T, Moen B, et al. Frequent musculoskeletal symptoms and reduced health-related quality of life among industrial workers. Occup Med 2002;52:91-8.

11 Leclerc A, Niedhammer I, Landre M-F, et al. One-year predictive factors for various aspects of neck disorders. Spine 1999;24:1455-62.

12 Fredriksson K, Alfredsson L, Köster M, et al. Risk factors for neck and upper limb disorders: results from 24 years of follow up. Occup Environ Med 1999;56:59-66.

13 Papageorgiou AC, Croft PR, Thomas E, et al. Influence of previous pain experience on the episode incidence of low back pain: results from the south Manchester back pain study. Pain 1996;66:181-5.

14 Cole DC, Hudak PL. Prognosis of nonspecific work-related musculoskeletal disorders of the neck and upper extremity. Am J Ind Med 1996:29:657-68.

15 Burdorf A, Naaktgeboren B, Post W. Prognostic factors for musculoskeletal sickness absence and return to work among welders and metal workers. Occup Environ Med 1998;55:490-5.

16 Turner JA, Franklin G, Turk DC. Predictors of chronic disability in injured workers: a systematic literature synthesis. Am J Ind Med 2000;38:707-22.

17 Crook J, Moldofsky. The clinical course of musculoskeletal pain in empirically derived groupings of injured workers. Pain 1996;67:427-33.

18 Waddell G. Epidemiology review: the epidemiology and cost of back pain. London: HMSO, 1994.

19 Ekberg K, Björkqvist B, Malm P, et al. Case-control study of the risk factors for disease in the neck and shoulders. Occup Environ Med 1994:51:262-6.

20 McCallum J, Shadbolt B. Self-rated health and survival: a 7-year follow-up study of Australian elderly. Am J Public Health 1994;84:1100-5.

21 Moller L, Kristensen TS, Hollnagel H. Self-rated health as a predictor of coronary heart disease. J Epidemiol Community Health 1996;50:423-8.

22 Ekberg K, Biörkqvist B, Malm P, et al. Cross-sectional study of risk factors for symptoms in the neck and shoulder area. Ergonomics 1995;38:971-80.

23 Waris P, Kourinka I, Kurppa K, et al. Epidemiologic screening of occupational neck and upper limb disorders: methods and criteria. Scand J Work Environ Health 1979;5:25-38.

24 Kourinka I, Jonsson B, Kilbom A, et al. Standardised Nordic questionnaire for the analysis of musculoskeletal symptoms. Appl Ergon 1987;18:233-7

25 Ware JE, Sherbourne CD. The MOS 36-item short-form health survey (SF-36). Med Care 1992;30:473-83.

26 Sullivan M, Karlsson J, Ware JE Jr. SF-36 Health survey. Swedish manual and interpretation guide. International Quality of Life Assessment (IQOLA) Project. Gothenburg, 1994 (in Swedish).

27 Estlander A-M, Takala E-P, Viikari-Juntura E. Do psychological factors predict changes in musculoskeletal pain? J Occup Environ Med 1998;40:445-53.

28 Manninen $\mathbf{P}$, Heliövaara $M$, Rihimäki $\mathrm{H}$, et al. Does psychological distress predict disability? Int J Epidemiol 1997;26:1063-70.

29 Blank N, Diderichsen F. The prediction of different experiences of longterm illness: a longitudinal approach in Sweden. J Epidemiol Community Health 1996:50:156-61.

30 Ohlsson K, Attewell RG, Johnsson B, et al. An assessment of neck and upper extremity disorders by questionnaire and clinical examination. Ergonomics 1994;37:891-7.

31 de Zwart BCH, Frings-Dresen MHW, Kihlbohm A. Gender differences in upper extremity musculoskeletal complaints in the working population. Int Arch Occup Environ Health $2001 ; 74: 21-30$. 